\title{
The meniscus depression of a porous spherical particle at the three phase contact line
}

\author{
P. Basařová ${ }^{1}$, D. Horn ${ }^{1}$ \& A. Capriotti ${ }^{2}$ \\ ${ }^{1}$ Department of Chemical Engineering, \\ Prague Institute of Chemical Technology, Czech Republic \\ ${ }^{2}$ Department of Chemical Engineering, \\ Mining and Environmental Technologies, University of Bologna, Italy
}

\begin{abstract}
The description of the processes at the three phase contact line is very important for the study of elementary steps in flotation. When a hydrophobic particle is attached to a gas-liquid interface, it is influenced by a number of forces, including surface tension, capillary and buoyancy forces on one hand and the particle weight on the other. In the case of porous particles, capillary forces acting in the porous surface must also be considered. The meniscus depression at the three-phase contact line is determined by the resultant force. The meniscus depression of the floating particle was studied using the image analysis method. The depression was studied for spherical polystyrene particles of diameters from 0.5 to $3 \mathrm{~mm}$. We compared the experimental values of meniscus depression with the data calculated using theoretical and semi-empirical models. The theoretical description of forces acting on the floating spherical porous particle is also given. We found that the existence of pores and surface inequalities has a significant influence on particle behaviour on the phase interface.
\end{abstract}

Keywords: floating particle, meniscus depression, porous surface, capillarity.

\section{Introduction}

The analysis of forces on the particle attached to the bubble surface has been the focus of the study of flotation processes since the early days of its industrial utilization. When a hydrophobic particle is attached to a gas-liquid interface, it is influenced by a number of forces, including the surface tension and buoyancy forces on the one hand and the particle weight on the other [1,2]. It is customary 
to sum the forces, which are a function of the position of the three-phase contact and to describe them jointly as the adhesive forces. The other forces, which are independent of the position of the three-phase contact, such as the particle weight less its buoyancy and the inertial forces, are jointly termed the detaching force.

One of the most important forces is the capillary force $F_{c}$. Due to the rotational symmetry along the vertical axis, the horizontal component of this force vanishes and only the vertical component remains.

$$
F_{c}=2 \pi r_{p} \sigma \sin \alpha \sin (\theta-\alpha)
$$

The next force, which supports the particle suspension at the interface, is the buoyancy $F_{b}$ of the particle immersed in the liquid phase. This force can be described by the following equation

$$
F_{b}=\frac{\pi r_{p}^{3} \rho_{l} g}{3}\left(2+3 \cos \alpha-\cos ^{3} \alpha\right)
$$

The pressure force $F_{p}$, which stabilizes the particle suspension, results from the hydrostatic pressure, which acts over an effective area $\pi r_{t p c}{ }^{2}$ enclosed by the three-phase contact line.

$$
F_{p}=\pi r_{p}^{2} H \rho_{l} g \sin ^{2} \alpha
$$

Another important force acting on the particle is the particle weight $F_{g}$, which tends to pull the attached particle into the liquid phase.

$$
F_{g}=-\frac{4 \pi r_{p}^{3} \rho_{p} g}{3}
$$

Here $\mathrm{H}$ is the meniscus depression at the three-phase contact, $r_{p}$ is the particle radius, $r_{\text {tcp }}$ is the radius of the three phase contact line, $\sigma$ is the surface tension, $g$ is the acceleration due to gravity, and $\rho_{\mathrm{p}}, \rho_{\mathrm{l}}$ and $\rho_{\mathrm{g}}$ are the densities of the particle, liquid and gas, respectively. $\theta$ is the contact angle and $\alpha$ is the central angle. The model behaviour of a solid particle is depicted on figure 1.

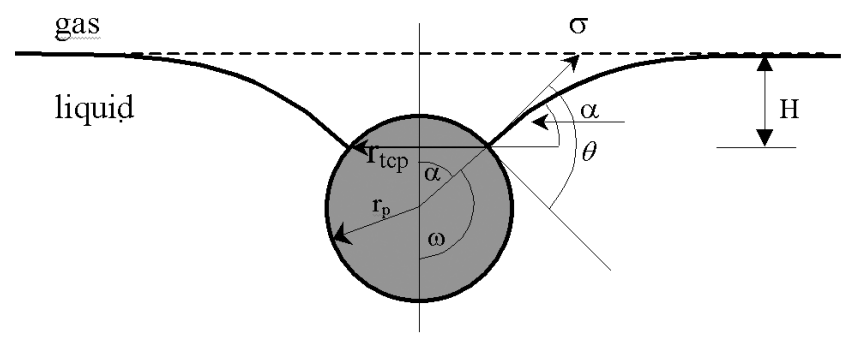

Figure 1: A model of solid spherical particle attached to planar gas-liquid surface.

In summary, there are four static forces acting on the smooth and spherical non-porous particle attached to a free water surface $[1,2,3]$. Equilibrium is attained if

$$
F_{c}+F_{b}+F_{p}+F_{w}=0
$$


and consequently

$$
\begin{aligned}
& \left\{\frac{H}{r_{p}} \sin ^{2} \alpha+\frac{2+3 \cos \alpha-\cos ^{3} \alpha}{3}\right\}\left(\rho_{l}-\rho_{g}\right) g \pi r_{p}^{3}+ \\
& 2 \pi r_{p} \sigma \sin \alpha \sin (\theta-\alpha)-g\left(\rho_{p}-\rho_{g}\right) 4 \pi r_{p}^{3} / 3=0
\end{aligned}
$$

Let's consider about a slightly porous particle. The capillary force for one pore [4] is given as

$$
F_{c}=2 \pi R_{p} \sigma \sin \gamma
$$

Here $R_{p}$ is the pore radius and $\gamma$ is the contact angle inside the pore. When we consider the capillary force for all pores around the three-phase contact line and the force equilibrium, it is possible to re-write equation 5 :

$$
F_{c}+F_{b}+F_{p}+F_{w}+\sum_{i} 2 \pi R_{p i} \sigma \sin \gamma_{i}=0
$$

This equation should be a general relationship to express the meniscus depression for a slightly porous particle.

In a flotation process, empirical equations are used often also. For three-phase contact line with small radius, the fundamental equation (6) can be solved by employing the method of matched asymptotic perturbation [5]. The final result for the meniscus depression is described by the Derjaguin equation:

$$
H=r_{t p c} \sin \beta\left(\ln \frac{4 L / r_{t p c}}{1+\cos \beta}-\gamma\right),
$$

where $\gamma=0.577$ and it is the Euler constant, $r_{\mathrm{tpc}}$ is the radius of three-phase contact line and due geometric symmetry $\beta=\theta-\alpha$ [2]. The capillary length $\mathrm{L}$ is defined by the following equation

$$
L=\sqrt{\frac{\sigma}{\left(\rho_{l}-\rho_{g}\right) g}} .
$$

Comparison of this equation to the numerical results of the Young-Laplace equation $^{2}$ indicates, that the Derjaguin equation is accurate for $r_{t c p} / L \leq 0.2$. For three-phase contact with large radius the approximate solution of the equation (6) can be derived also. For the meniscus depression the modification yields

$$
\frac{H}{L}=\sin (\beta / 2) \frac{2}{\sqrt{1+\left(L / r_{t p c}\right)}}
$$

This simple equation derived in the limit of the large radius compares with the numerical results surprisingly well, down to $r_{t c p} / L \geq 2$. For three-phase contacts with the radius in the range of $2.0 \geq r_{t c p} / L \geq 0.2$ no analytical solutions for the meniscus depression are available. The explicit dependence of the meniscus depression on the three-phase contact radius and the meniscus angular inclination can be obtained only empirically. Nguyen [3] describes this solution based on the asymptotic analytical results and the numerical 
computational results of solution to the Young-Laplace equation. According to this method one can obtain

$$
\frac{H}{L}=a \sin (b \beta),
$$

where $\mathrm{a}$ and $\mathrm{b}$ are empirical parameters. It is assumed, that $\mathrm{b}$ is independent of $\beta$ and is a function of $r_{t c p} / L$ only. It has two asymptotes, $\mathrm{b}=0.5$ and 1 for very small and very large values of $r_{t c p} / L$, respectively. For the range of $2 \geq r_{t c p} / L \geq 0.1$, fitting to the exact numerical data gives:

$$
b=0.5+0.332 \exp \left(-2.122 r_{t p c} / L\right) \quad \text { and } \quad a=\frac{2.186}{1+0.649\left(L / r_{t p c}\right)}
$$

The aim of this research was the experimental study of the meniscus depression at the three-phase contact of the floating plastic particle using image analysis method. Plastic materials are characteristic for their polymeric structure with long chains. Therefore for these compounds it is possible to assume the occurrence of surface inequality from a microscopic point of view and also the appearance of pores. The depression was studied for polystyrene particles of different diameters $(0.1-3 \mathrm{~mm})$. All experiments were performed in a small apparatus monitored by a high-speed CCD camera with a macro-objective. The obtained data were processed using an image analysis software LUCIA. The experimental data were compared with the theoretical models.

\section{Experimental materials and methods}

The model spherical particles of expandable polystyrene EPS (originating from Kaucuk Kralupy, Czech. Rep.) were used for the experiments. We used 70 particles with diameters from $0.5 \mathrm{~mm}$ to $2.6 \mathrm{~mm}$ and the density of these balls was $1.03 \mathrm{~g} . \mathrm{cm}^{-3}$. The value of the contact angle for this type of polystyrene in distilled water was $79,2^{0}$ [6]. The contact angles were measured by direct method, monitoring the drop profile on smooth flat surface, represented by a foil made from polystyrene balls. For all the measurements the distilled and deionised water was used. Here, $\mathrm{pH}$ was 6.13 and conductivity $1,6 \mu \mathrm{S} / \mathrm{cm}$.

For interpretation of obtained data the program LUCIA was used. LUCIA is a software program evaluated for capturing, analysing and saving images. This program works with an A/D converter on the graphical chip in computer, the graphical chip converts the analogue signal to the digital signal. The program uses dimensional analysis and with a calibrated picture it is possible to obtain the precise size of particles. The program enables automatic detection of boundaries, measurement of angles and automatic calculation of the count of objects with help of attributes of objects. The system of image analysis consists of three parts: a microscope, CCD camera and PC with LUCIA software for analysis of captured images. Resulting images from the experiment are adjusted by the software (brightness, contrast and detection of objects) and mathematically evaluated. 


\section{Results and discussions}

For polymeric compounds it is possible to assume the occurrence of surface inequality from a microscopic point of view and also the appearance of pores. As an experimental material we used expandable polystyrene, which contains pentane as an expanding component. This gas vanishes after a short time, but in the interior structure small bubbles or holes remain. Their diameter is $6 \mu \mathrm{m}$ and this value was measured using the electronic microscope. If the bubble is in the near vicinity of the surface, it can happen that the smooth structure is damaged and a pore is originated. On the figure 2 are illustrated details of the surface with a big enlargement. Here some pores and surface inequalities are visible. From the image analysis we know that the pore radii lie between 6 to $15 \mu \mathrm{m}$. Unfortunately, it was not possible to measure pore diameters for all particles separately. According to our measurements we expect that the number of pores could not exceed the value of 5 pores per $1 \mathrm{~mm}$ on the contact line.

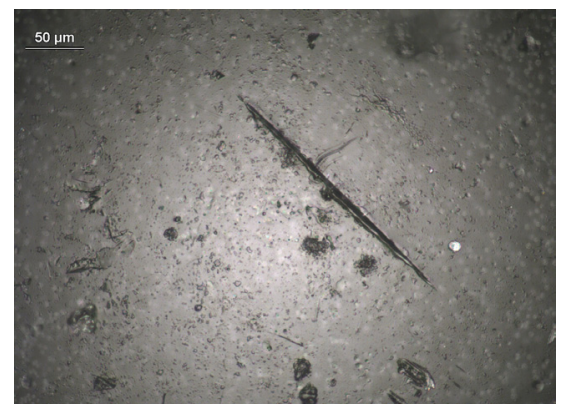

Figure 2: $\quad$ The detail of a surface.

The images of floating particles were the basis of all experimental data. The software LUCIA uses dimensional analysis and using calibrated images the particle diameters were determined with an accuracy $0,001 \mathrm{~mm}$. The obtained data $\left(r_{p}\right)$ were utilized for the assessment of the radius of the three-phase contact $\mathrm{r}_{\mathrm{tpc}}$ and meniscus depression $\mathrm{H}$. On the figure 3 (left) is an original image of a floating particle. The scanned image was processed using the software LUCIA On a figure 3 (right) is plotted a spherical particle shape and results of a size analysis are also marked.

All used experimental particles have the same density and the value of a contact angle. Therefore, it is possible to presume, that the radius of three-phase contact $r_{\text {tpc }}$ should be proportional to the particle radius $r_{p}$. This dependence was confirmed and the results are depicted on the figure 4 . We found, that the radius of three-phase contact can be calculated for the polystyrene balls using the equation

$$
r_{t p c}=0,8835 r_{p}
$$



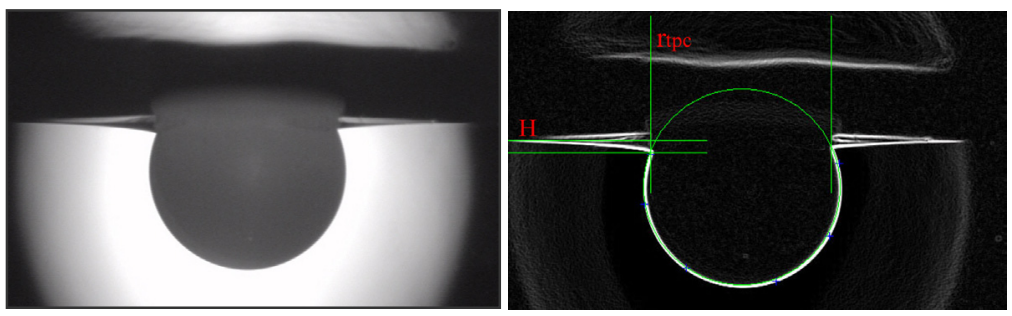

Figure 3: Detail of the floating particle (left) and the image analysis using the software LUCIA (right).

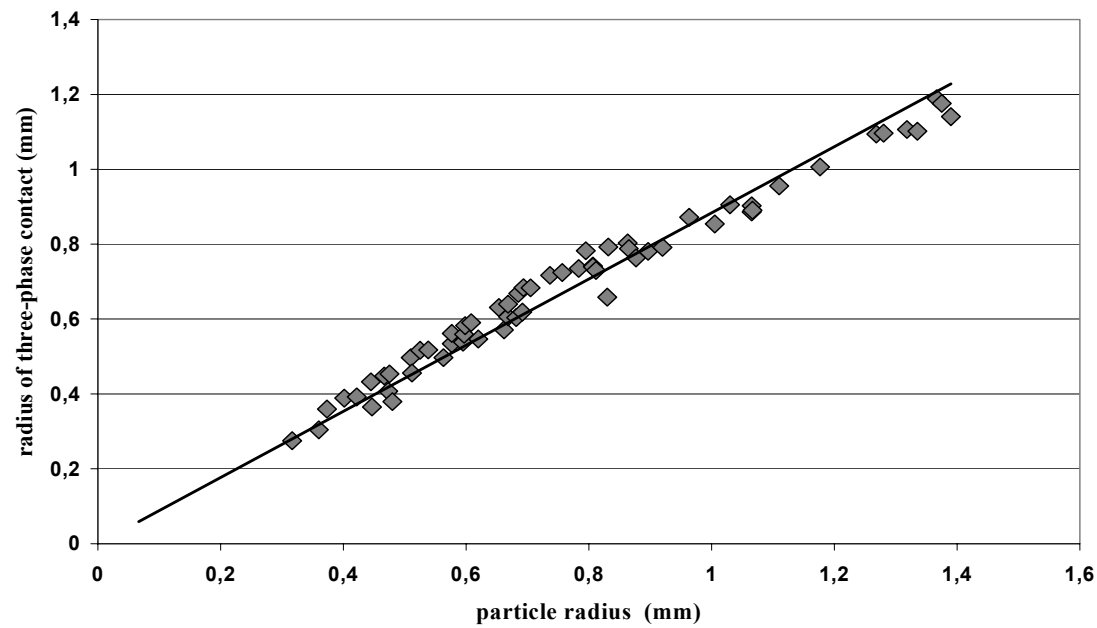

Figure 4: The dependence of the radius of the three-phase contact $r_{\text {tpc }}$ on the particle radius $r_{p}$.

The angle $\alpha$ was measured experimentally and the angle $\beta$ (see Fig.1) was calculated according to the relation $\beta=\theta-\alpha$ [2]. For the calculation of meniscus depression $H$ three equations were used. In the literature [2,3], it is recommended to calculate meniscus depression with regard to the value of $r_{\text {tcp }} /$ L. For three-phase contact with small radius $\left(r_{t c p} / L \leq 0.2\right)$ the Derjaguin equation (9) is suggested. The data, calculated using this equation, are denoted as $\mathrm{H}_{\text {small }}$. For three-phase contacts with the radius in the range of $2.0 \geq r_{\text {tcp }} / L \geq 0.2$ the semi-theoretical equations (12-13) were used. The calculated values are denoted as $\mathrm{H}_{\text {middle. Finally the fundamental theoretical }}$ equation (6) was applied. The calculated data are denoted as $\mathrm{H}_{\text {theor }}$. On figure 5 are demonstrated experimental data and calculated dependences of meniscus depression on the radius of three-phase contact. 


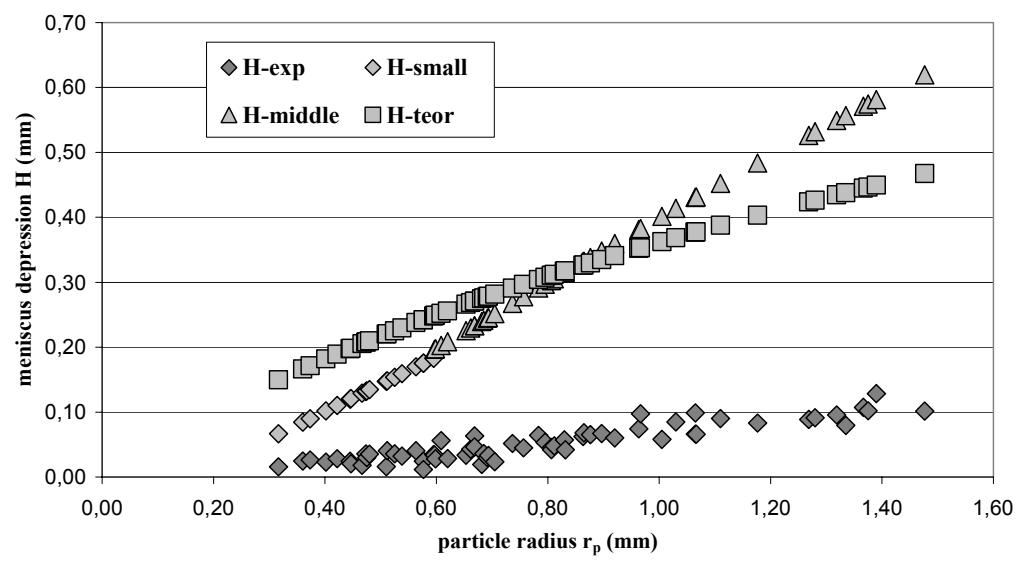

Figure 5: The dependence of the meniscus depression on the radius of threephase contact line - experimental and calculated data.

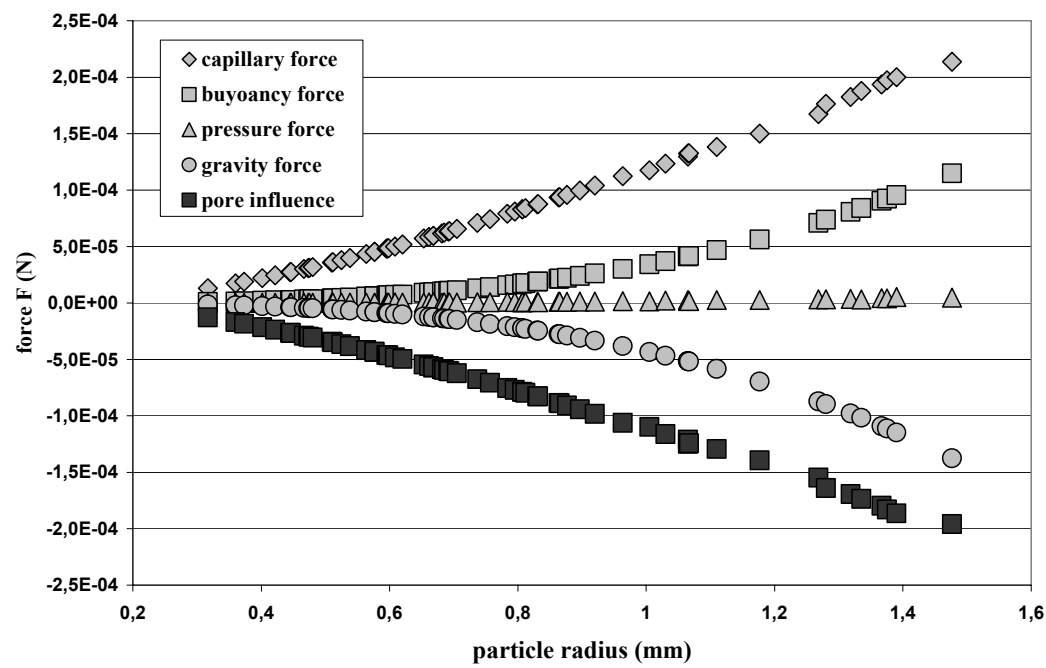

Figure 6: The calculated values of capillary force $F_{c}$, buoyancy $F_{b}$, pressure force $F_{p}$, particle weight $F_{g}$ and capillary force of pores $F_{c}$ and their dependence on the particle radius.

A substantial inconsistency of experimental and calculated data is obvious from these results. All used theoretical relationships (equations 6, 9 and 12-13) were derived for smooth and non-porous spherical particles and therefore do not reflect the pore existence. It is obvious from experimental measurements that the influence of surface roughness and pores is remarkable. The value of meniscus depression is even several times lower. Thus for even slightly porous particles 
the usage of theoretical relationships is not suitable for calculations in flotation, e.g. for the estimation of maximum size of a floating particle.

On figure 6 are plotted the calculated values of capillary force $F_{c}$, buoyancy $F_{b}$, pressure force $F_{p}$ and particle weight $F_{g}$ as a dependence on the particle radius. Also the force reflecting the pore influence was calculated according to eqn. (8). Here the force balance at equilibrium should be equal to zero. The most significant forces are the capillary force acting on the whole particle and the force influenced by the pore existence. This result is not surprising if we consider all aspects of the law of capillary rise [4]. On figure 7 is given one example of the estimation of pore number. Here the pore radius $15 \mu \mathrm{m}$ was used and the calculation was done for several values of the contact angle. We could expect that the contact angles in pores have the same or lower value than the contact angles on a flat surface.

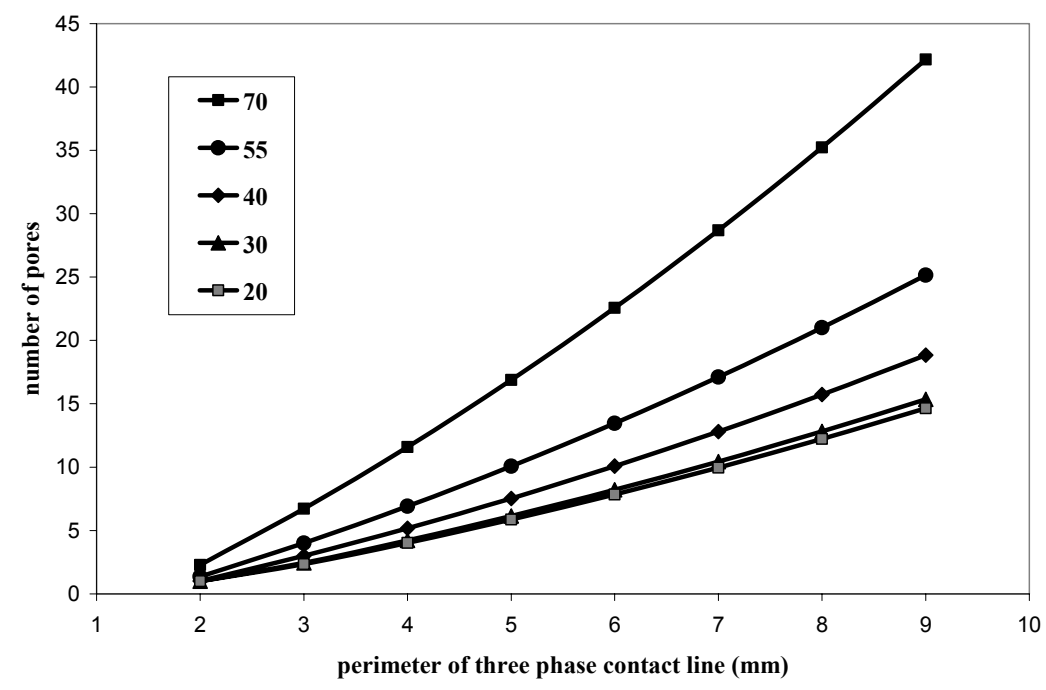

Figure 7: The estimated number of pores as a dependence on the perimeter of the three phase contact line for contact angles from $20^{\circ}$ to $70^{\circ}$ and pore radius $15 \mu \mathrm{m}$.

\section{Conclusions}

The meniscus depression $\mathrm{H}$ at the three-phase contact line of the floating slightly porous particle was studied using the image analysis method (software LUCIA). We compared the experimental values of meniscus depression with the data calculated using theoretical and semi-empirical models derived for the nonporous particles. We found out a big difference between experimental and theoretical values. It is obvious from experimental measurements that the influence of surface roughness and pores is remarkable. The value of meniscus 
depression is even several times lower. Thus for even slightly porous particles the usage of theoretical relationships is not suitable for calculations in flotation, e.g. for the estimation of maximum size of a floating particle. The existence of pores and surface inequalities has a really significant influence on the particle behavior on the phase interface.

\section{Acknowledgements}

This work has been supported by the Grant no. 104/05/2566 from the Grant Agency of Czech Rep. and by the Grant Research Project MSM60446137306 of the Czech Ministry of Education.

\section{References}

[1] Schulze, H.J., Physico-chemical Elementary Processes in Flotation, Elsevier: Amsterdam, Oxford, New York and Tokyo, pp.182-196, 1984.

[2] Nguyen, A. V.\& Schulze, H .J., Colloidal Science of Flotation, Surfactant Science Series, Marcel Dekker: New York and Basel, pp.527-558, 2004.

[3] Nguyen, A.V., Empirical equations for meniscus depression by particle attachment. J. Colloid Interface Sci. 249, pp. 147-151, 2002.

[4] De Gennes, P.G., Quere, D., Brochard-Wyart, F. \& Reisinger, A., Capillarity and Wetting Phenomena: Drops, Bubbles, Pearls, Waves, Springer-Verlag: New York, pp. 51-53, 2004.

[5] Derjaguin, B., Theory of meniscus depression in liquids due to immersed small objects and its utilization for the measurements of border contact angles for thin threads and fibres. Dokl. Akad. Nauk SSSR 51(7), pp. 517520, 1946.

[6] Basařová, P. \& Horn, D., Study of the hydrodynamics in the plastics flotation, Proc. of $50^{\text {th }}$ Conf. of Chem. and Proc. Engineering - CHISA 2003, eds. J. Novosad, Praha, pp.1-7, 2003. 